Gutiérrez-Castañón, E.; Martínez-de-Haro, V.; Ramos-Álvarez, J.J. y Cid-Yagüe, L. (2018) Lesiones en Educación Física de Educación Secundaria ¿Un problema? / Injuries in Physical Education of High School. A Problem? Revista Internacional de Medicina y Ciencias de la $\begin{array}{lllllll}\text { Actividad Física y el Deporte vol. } 18 & \text { (72) pp. 709-722 }\end{array}$ Http://cdeporte.rediris.es/revista/revista72/artepidemiologia965.htm

DOI: http://doi.org/10.15366/rimcafd2018.72.007

\title{
ORIGINAL
}

\section{LESIONES EN EDUCACIÓN FÍSICA DE EDUCACIÓN SECUNDARIA ¿UN PROBLEMA?}

\section{INJURIES IN PHYSICAL EDUCATION OF HIGH SCHOOL. A PROBLEM?}

\author{
Gutiérrez-Castañón, E. ${ }^{1}$; Martínez-de-Haro, V.'; Ramos-Álvarez, J.J. ${ }^{3}$ y Cid- \\ Yagüe, L. ${ }^{4}$ \\ ${ }^{1}$ Doctor en Ciencias de la Actividad Física y el Deporte. CEIP San Carlos, Madrid (España) \\ eliasguticas@hotmail.com \\ 2 Doctor en Medicina y Cirugía. Departamento de Educación Física, Deporte y Motricidad \\ Humana. Facultad de Formación del Profesorado y Educación. Universidad Autónoma de Madrid \\ (España) vicente.martinez@uam.es \\ ${ }^{3}$ Doctor en Medicina y Cirugía. Especialista en Medicina del Deporte. Escuela de Medicina de la \\ Educación Física y el Deporte. Universidad Complutense de Madrid (España) \\ jjramosa@med.ucm.es \\ ${ }^{4}$ Doctora en Ciencias de la Actividad Física y el Deporte. Departamento de Educación Física, \\ Deporte y Motricidad Humana. Facultad de Formación del Profesorado y Educación. Universidad \\ Autónoma de Madrid (España) lourdes.cid@uam.es
}

Código UNESCO / UNESCO Code: 3299 Medicina del deporte / Sport Medicine. Clasificación del Consejo de Europa / Council of Europe classification: 11 Medicina del deporte / Sport Medicine.

Recibido 3 de diciembre de 2016 Received December 3, 2016 Aceptado 17 febrero de 2017 Accepted February 17, 2017

\section{RESUMEN}

El control de la incidencia de lesiones en la clase de Educación Física debe ser un factor prioritario para la salud pública. El objetivo de este estudio es analizar la incidencia de lesiones en las clases de Educación Física (EF) y cómo se relacionan con la falta de participación de los estudiantes. Estudio de cohortes prospectivo. Conglomerado de muestreo bi-etapa para un total de 637 estudiantes. Las ausencias y las lesiones se registraron durante el curso escolar. De 1.463 sesiones, solo el 4,58\% registró ausencias causadas por lesión (0,33\% de la participación total esperada). La tasa de lesiones producidas en las clases de Educación Física fue de 1,90 por cada 1.000 horas de participación. La frecuencia de ausencia en las clases de Educación Física debido a una lesión 
es muy baja en comparación con la participación general esperada; por lo tanto, no es un factor que influya significativamente en la tasa de participación de los estudiantes.

PALABRAS CLAVE: Lesión, Educación Física, Adolescente, Educación secundaria.

\section{ABSTRACT}

The incidence of injuries in physical education class should be a priority factor for public health. The aim of this study is to analyze the incidence of injuries in Physical Education (PE) lessons and how they relate to lack of full student participation. Prospective cohort study. Conglomerate bi-stage sampling for a total of 637 students. Absence and injuries were recorded for the school year. Of 1463 lessons, only $4.58 \%$ were documented with identification of the causal incident $(0.33 \%$ of the total participation expected). The rate of injuries produced in PE lessons was of 1.90 for every 1000 hours of participation. The frequency of absence of in PE lessons due to injury is very low compared to the overall expected participation; therefore it is not a factor that influences the rate of student participation significantly.

KEY WORDS: Injury, Physical education, Adolescent. High school. 


\section{INTRODUCCIÓN}

No existe unanimidad sobre la relevancia de las lesiones producidas durante la clase de Educación Física (EF). Algunos autores consideran las lesiones escolares un factor prioritario para la salud pública (Taylor y Attia, 2000) al incidir en el proceso educativo (Sosnowska \& Kostka, 2003) y convertirse en un problema económico grave derivado de costes sanitarios, pérdida de días de trabajo, etc. (Abernethy y MacAuley, 2003; Collard, Verhagen, van Mechelen, Heymans, y Chinapaw, 2011), mientras otros autores consideran que las lesiones deportivas durante los años escolares no son tan relevantes (Grimaud, Piette, Clappier, Deguen y Pommier, 2007) y tienen efectos mínimos a medio y largo plazo.

Los estudios específicos de lesiones en la clase de Educación Física son muy escasos y, habitualmente, hacen referencia a lesiones en general, sin especificar las producidas durante la clase de Educación Física. Esto lleva a una confusión de términos como deporte escolar, educación física y ejercicio físico, que luego complica el análisis de los datos y la identificación de la causa real de las lesiones.

Múltiples estudios recogen datos de registros hospitalarios, clínicas deportivas y centros pediátricos sobre lesiones deportivas generales, catalogándolas como escolares tanto por originarse en actividades relacionadas con la escuela como por producirse en edad escolar (Belechri, Petridou, Kedikoglou, Trichopoulos, \& Sports Injuries Unión Europea, 2001; Habelt, Hasler, Steinbruck y Majewski, 2011; Loder \& Abrams, 2011; Pakzad-Vaezi \& Singhal, 2011; Roach \& Maffulli, 2003). Otros recogen datos en la escuela o actividades relacionadas con ella, mencionando las relacionadas con la actividad física, pero sin diferenciar entre las distintas actividades que se realizan dentro de la escuela (Lenaway, Ambler y Beaudoin, 1992; Spinks, Macpherson, Bain y McClure, 2006). Los estudios que se centran en la actividad física o los deportes escolares no establecen registros diferenciados para las clases de Educación Física (Emery, 2010; Josse, MacKay, Osmond y MacPherson, 2009).

Finalmente, los pocos estudios que se centran específicamente en la EF difieren ampliamente en sus metodologías (Backx, Beijer, Bol y Erich, 1991; Carmeli, Azencot, Wertheim y Coleman, 2003; Collard, Chinapaw, van Mechelen, y Verhagen, 2009; Sundblad, Saartok, Engstrom y Renstrom, 2005; Videmsek, Karpljuk, Mlinar, Mesko y Stihec, 2010), por lo que la interpretación de sus resultados debe realizarse con cautela. Por un lado, tienen diferentes tamaños de muestra que van desde amplias regiones geográficas (Nelson, Alhajj, Yard, Comstock y McKenzie, 2009) hasta estudios de un único centro escolar, diferencias en los períodos de tiempo, con datos registrados durante 1315 años (Pakzad- Vaezi \& Singhal, 2011) frente a algunos reunidos en un único mes (Grimaud et al., 2007), y adicionalmente, grandes diferencias en los grupos de edad de las poblaciones estudiadas, que van de 5 a 19 años de edad (Carmeli et al., 2003; de Loes, Jacobsson, \& Goldie, 1990; Nelson et al., 2009; Spinks et al., 2006; Verhagen, Collard, Paw, y van Mechelen, 2009). Por otro lado, encontramos diferencias en los instrumentos utilizados, existiendo mediciones estandarizadas como NEISS en los Estados Unidos (Nelson et al., 2009) y 
CHRIPP en Canadá (Pakzad-Vaezi \& Singhal, 2011), con gran cantidad de datos, de rápida clasificación y acceso pero basados en ingresos hospitalarios y atención en urgencias e inevitablemente sesgados hacia las lesiones más graves; $y$ estudios basados en encuestas realizadas en la escuela (Emery, 2010; Sundblad et al., 2005), siendo en muchos casos, una combinación de registros de incidencias y encuestas sobre hábitos deportivos (Verhagen et al., 2009) 0 diarios de actividades de tiempo libre (Spinks et al., 2006). A estos se añaden nuestros estudios (Gutiérrez-Castañón, E, 2014, 2008; Gutiérrez-Castañón, E et al., 2007).

Las clasificaciones internacionales de referencia, como NOMESCONCECI (NOMESCO, 2007) y la Clasificación Internacional de Causas Externas de Lesiones (McKenzie, Fingerhut, Walker, Harrison, \& Harrison, 2012) muestran una gran imprecisión al determinar las actividades en el momento de producirse la lesión, agrupando bajo un mismo epígrafe las clases de Educación Física y el deporte relacionado con la escuela. A esto se añaden otros dos inconvenientes, citados por algunos autores (Carter, Westerman, \& Hunting, 2011). En primer lugar, el cálculo de la tasa de lesiones en una población de referencia puede incluir a no participantes en la población total de una región o escuela. Este problema se trata de resolver incluyendo la variable de exposición del atleta (AE), sin embargo, aún incluiría períodos sin exposición al riesgo. En segundo lugar, la dificultad de determinar con exactitud el tiempo de exposición al riesgo. Esto se trata de resolver a través de encuestas de actividad física y hábitos deportivos de tiempo libre, para al menos conseguir una estimación aproximada del tiempo de exposición al riesgo, pero sigue siendo imprecisa (Spinks et al., 2006; Verhagen et al., 2009).

Por lo tanto, teniendo en cuenta la gran variabilidad de los resultados obtenidos previamente y la diversidad de métodos empleados, el presente artículo tiene como objetivo obtener registros que muestren, de la manera más objetiva posible, la incidencia real de lesiones durante las clases de Educación Física. Para este propósito, se proponen los siguientes objetivos: 1. Conocer la incidencia de lesiones durante las clases de Educación Física que evitan que los estudiantes participen activamente en clase, en comparación con otros problemas de salud no relacionados con lesiones. 2. Analizar la naturaleza de las lesiones producidas en las clases de EF, su distribución anatómica, su mecanismo de producción y el tratamiento seguido para su recuperación.

\section{MÉTODOLOGÍA}

La muestra incluyó 26 grupos de clase de $4^{\circ}$ curso de Educación Secundaria de centros educativos de la Comunidad de Madrid (España), para un total de 637 alumnos con edades comprendidas entre 15 y 17 años, que corresponden al último año de educación obligatoria.

Para seleccionar los grupos se utilizó un muestreo por conglomerados bietápico con selección aleatoria en cada etapa. La primera etapa con selección aleatoria entre todos los centros de Educación Secundaria Obligatoria de la Comunidad de Madrid y la segunda etapa seleccionando un grupo al azar de todos los grupos de cuarto curso disponibles en los centros elegidos 
previamente. Los registros de participación y ausencia fueron revisados diariamente por los profesores de cada grupo.

Las clases de Educación Física duran una hora, el tiempo de actividad se calculó sobre el total de horas.

La ausencia en las clases de educación física se registró durante todo el curso escolar (desde la tercera semana de septiembre hasta la segunda semana de junio). Estos datos fueron recogidos por los profesores de EF de cada Centro, tras una reunión e instrucción previa con los autores. Los criterios utilizados para determinar la falta de participación o ausencia de los estudiantes fueron los siguientes:

- La participación fue considerada como la presencia activa de un alumno en cada clase de Educación Física, diferente a la asistencia que solo requiere la presencia del alumno sin un compromiso activo. El total de participaciones esperadas se corresponde con el producto del número de alumnos de cada grupo por el número de sesiones de Educación Física de dicho grupo

- Se consideró falta de participación o ausencia el hecho de que el alumno no participara por completo durante la clase de Educación Física, incluyendo tanto situaciones en las que no estaba presente, como aquellas situaciones en las que, a pesar de estar presente, el alumno no participó con el resto del grupo.

Las causas de cada ausencia se clasificaron en función de los siguientes criterios:

- La falta general de participación. Se diferenció entre faltas por motivos de salud y por otros motivos (incluidas sanciones disciplinarias, falta de equipo adecuado, absentismo, etc.).

- La ausencia relacionada con la salud. Diferenciando entre faltas de actividad por enfermedad y por lesión.

- Aquellas ausencias registradas como originadas por lesiones fueron divididas en lesiones producidas durante la clase de Educación Física y lesiones que ocurrieron fuera de Educación Física.

Esto dio como resultado cinco categorías posibles de participación: participación normal, falta de participación debida a enfermedad, falta de participación debida a una lesión en Educación Física, falta de participación debida a una lesión fuera de Educación Física y falta de participación debida a otros motivos.

Los autores personalmente recopilaron las lesiones utilizando una hoja de registro que refleja las categorías previamente establecidas e incluye apartados para observaciones del investigador que realiza la recogida de datos, agregando preguntas relacionadas con la actividad física durante el tiempo libre y la frecuencia semanal de cada una, clasificadas como actividad física libre y 
organizada, y una estimación del número de horas dedicadas a estas actividades.

Las lesiones se definieron y clasificaron según el Modelo de seguridad del fútbol de la UEFA (Hagglund, Walden, Bahr y Ekstrand, 2005) y el Grupo de consenso sobre lesiones de la FIFA (Fuller et al., 2006). El tipo de lesión se clasificó de acuerdo con el tipo de mecanismo que la produjo: traumatismo directo (conmociones cerebrales, fracturas), traumatismo indirecto (esguinces, lesión muscular de diverso grado) y lesiones por uso excesivo o sobrecarga (tendinopatía, condropatía, etc.).

El software SPSS 20.0 fue utilizado para procesar los datos. Se realizaron recuentos de frecuencia para toda la muestra y los subgrupos establecidos en cada sección del estudio, de acuerdo con las causas de la falta de participación de los estudiantes. Las pruebas de contraste de las pruebas de frecuencia y bondad de ajuste también se llevaron a cabo utilizando un análisis de chicuadrado con un nivel de significación de 0,05.

\section{RESULTADOS}

Del total de la muestra, 335 estudiantes (52,6\%) eran varones y 302 $(47,4 \%)$ mujeres, con un total de 52 estudiantes $(8,16 \%)$ que no presentaron ninguna falta de participación a lo largo el estudio y 241 (37,84\%) que registraron alguna falta de participación por lesión, con 64 de ellos $(10,04 \%)$ sufriéndola como consecuencia del desarrollo de la clase de Educación Física. El número de sesiones analizadas fue de 1.463 sesiones, con 67 de ellas registrando un incidente de lesión durante su desarrollo (4,58\%) mientras que el número total de participaciones esperadas ascendió a 35.191.

Estos datos corresponden a una tasa de lesiones de 1,90 por cada 1.000 horas de exposición a clases de Educación Física. Por otro lado, la tasa de lesiones fuera de las clases de Educación Física fue 2,09 por cada 1.000 horas de actividad física organizada y 2,13 por cada 1.000 horas de actividad física no organizada.

\section{Participación y ausencia en las clases de educación física}

La Figura 1 resume las participaciones totales y las ausencias que se registraron en la clase de Educación Física en relación con las causas que las originaron. 


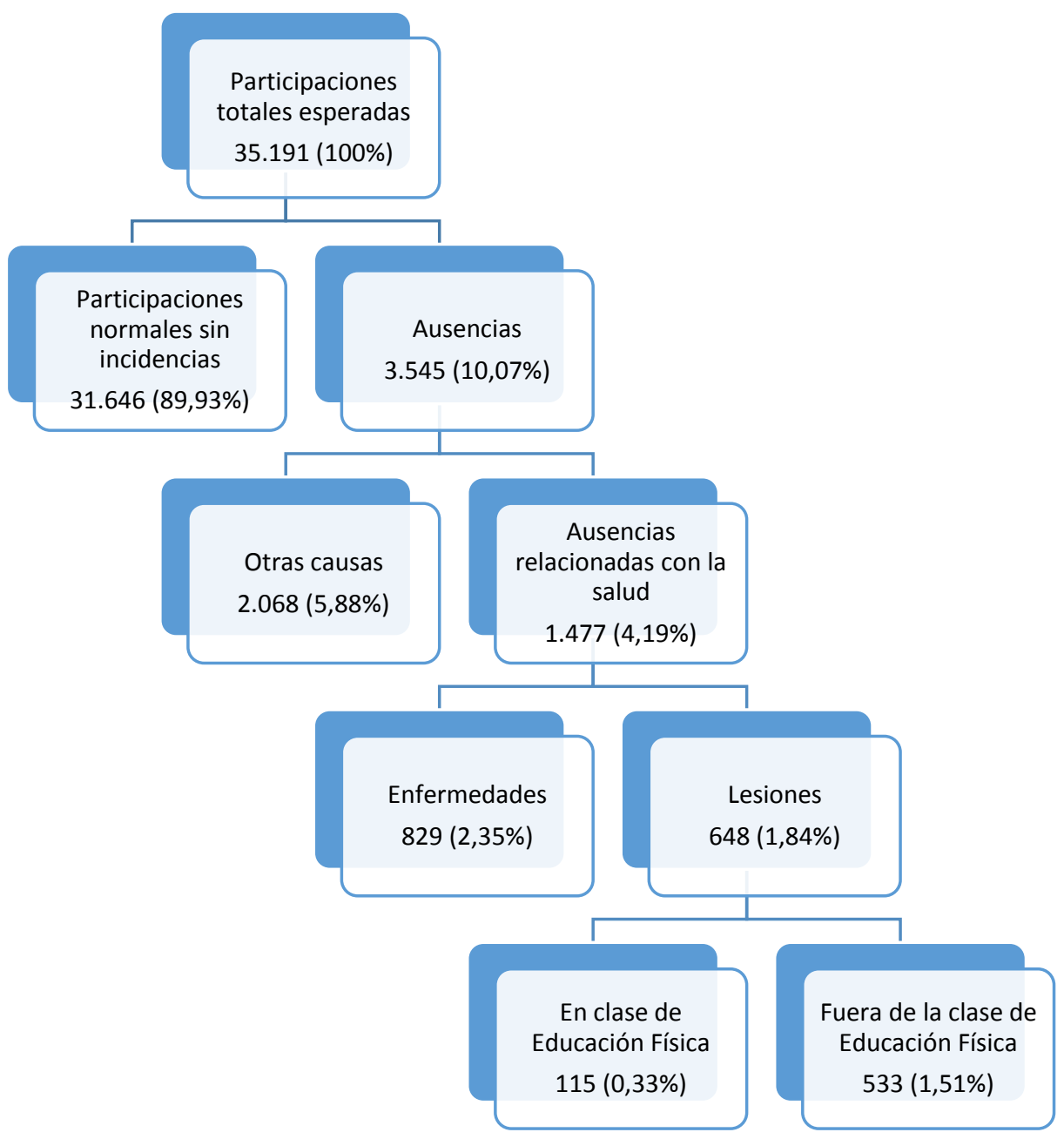

Figura 1. Participaciones y ausencias según la causa

\section{Tipos de lesiones registradas}

La figura 2 muestra el tipo de lesiones que se originaron en las clases de educación física y las ausencias que produjeron. 


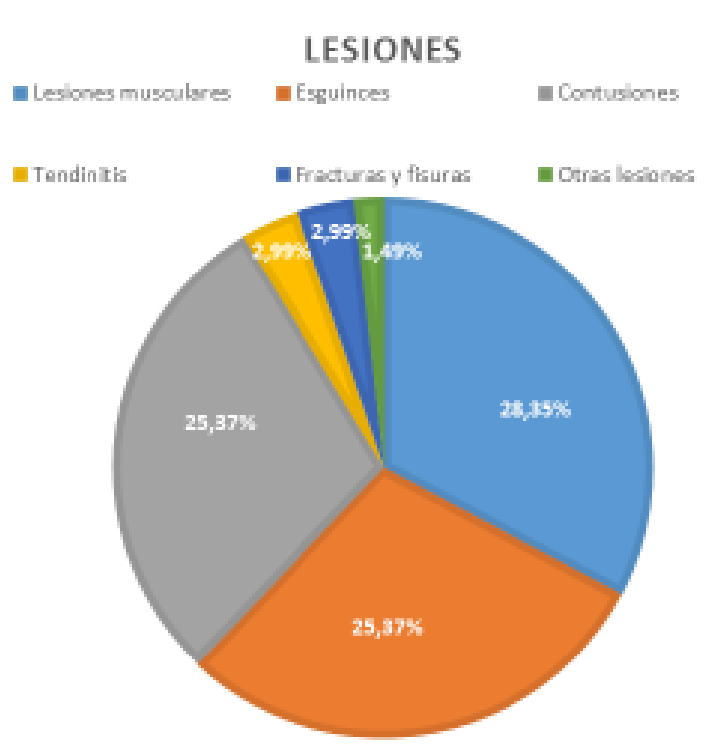

\section{AUSENCIAS CAUSADAS POR ESTAS LESIONES}

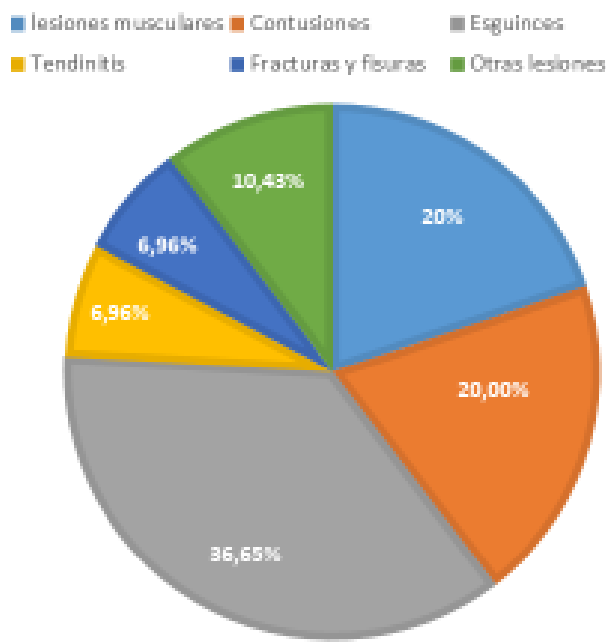

Figura 2. Lesiones ocurridas en clase y ausencias que producen.

\section{Lesiones y ausencia según región anatómica, mecanismo de producción y tratamiento recibido}

Dada la baja incidencia de lesiones registradas, solo se consideraron tres regiones anatómicas: cabeza y tronco, extremidades superiores y extremidades inferiores (Tabla 1).

\begin{tabular}{|l|c|c|c|c|}
\hline \multirow{2}{*}{\multicolumn{1}{c}{ Región anatómica }} & \multicolumn{2}{c|}{ Lesiones } & \multicolumn{2}{c|}{ Ausencias } \\
\cline { 2 - 5 } & $\mathrm{n}$ & $\%$ & $\mathrm{n}$ & $\%$ \\
\hline Cabeza y tronco & 4 & $5,97 \%$ & 6 & $5,22 \%$ \\
\hline Extremidades superiores & 14 & $20,90 \%$ & 26 & $22,61 \%$ \\
\hline Extremidades inferiores & 49 & $73,13 \%$ & 83 & $72,17 \%$ \\
\hline Total & 67 & $100 \%$ & 115 & $100 \%$ \\
\hline
\end{tabular}

\begin{tabular}{|l|c|c|c|}
\hline & Chi-cuadrado & df & Sig. asintótica \\
\hline Ajuste de lesiones & 50,000 & 2 & 0,000 \\
\hline Ajuste de lesiones -Participación & 0,292 & 2 & 0,864 \\
\hline
\end{tabular}

Tabla 1. Lesiones y faltas de participación con origen en la clase de EF agrupadas por región anatómica. Resultados de test de bondad de ajuste según distribución homogénea y en relación lesiones-participaciones.

En la Tabla 2, todas las lesiones se clasifican de acuerdo con el mecanismo por el cual se produjeron. Con respecto al tratamiento recibido, solo se consideraron dos situaciones, incluida la atención médica (médico, enfermera o fisioterapeuta) o la ausencia de tratamiento (Tabla 3). 


\begin{tabular}{|l|c|c|c|c|}
\hline \multirow{2}{*}{$\begin{array}{c}\text { Mecanismo } \\
\text { de lesión }\end{array}$} & \multicolumn{2}{c|}{ Lesiones } & \multicolumn{2}{c|}{ Ausencias } \\
\cline { 2 - 5 } & $\mathrm{n}$ & $\%$ & $\mathrm{n}$ & $\%$ \\
\hline Traumatismo directo & 19 & $28,36 \%$ & 31 & $29,96 \%$ \\
\hline Traumatismo indirecto & 32 & $47,76 \%$ & 59 & $51,30 \%$ \\
\hline Sobrecarga & 16 & $23,88 \%$ & 25 & $21,74 \%$ \\
\hline Total & 67 & $100 \%$ & 115 & $100 \%$ \\
\hline
\end{tabular}

\begin{tabular}{|l|c|c|c|}
\hline & Chi-cuadrado & df & Sig. asintótica \\
\hline Ajuste de lesiones & 6,478 & 2 & 0,039 \\
\hline Ajuste de lesiones -Participación & 0,605 & 2 & 0,739 \\
\hline
\end{tabular}

Tabla 2. Lesiones y faltas de participación con origen en clase de E.F. agrupadas por mecanismo de producción. Resultados de test de bondad de ajuste según distribución homogénea y en relación lesiones-participaciones.

\begin{tabular}{|l|c|c|c|c|}
\hline \multirow{2}{*}{$\begin{array}{c}\text { Tratamiento } \\
\text { recibido }\end{array}$} & \multicolumn{2}{|c|}{ Lesiones } & \multicolumn{3}{c|}{ Ausencias } \\
\cline { 2 - 5 } & $\mathrm{n}$ & $\%$ & $\mathrm{n}$ & $\%$ \\
\hline Sin tratamiento & 40 & $59,70 \%$ & 46 & $40 \%$ \\
\hline Tratamiento médico & 27 & $40,30 \%$ & 69 & $60 \%$ \\
\hline Total & 67 & $100 \%$ & 115 & $100 \%$ \\
\hline
\end{tabular}

\begin{tabular}{|l|c|c|c|}
\hline & Chi-cuadrado & df & Sig. asintótica \\
\hline Ajuste de lesiones & 2,522 & 1 & 0,112 \\
\hline Ajuste de lesiones -Participación & 18,550 & 1 & 0,000 \\
\hline
\end{tabular}

Tabla 3. Lesiones y faltas de participación agrupadas por tratamiento posterior recibido. Resultados de test de bondad de ajuste según distribución homogénea y en relación lesionesparticipaciones.

\section{DISCUSIÓN}

La tasa de ausencia en la clase de Educación Física resultó bastante baja (por debajo del $0,4 \%$ ) en comparación con los datos de participación. Los resultados de este estudio coinciden con los realizados en períodos más cortos en el hecho de que la incidencia de lesiones en las clases de EF parece menor que las lesiones producidas en otros tipos de actividad física (Abernethy y MacAuley, 2003; Sosnowska y Kostka, 2003). Debido a la gran variabilidad en los métodos de recopilación de datos y tamaños de muestra, todavía no hay unanimidad en cuanto a la tasa de lesiones y el tiempo de exposición al riesgo.

El presente estudio solo consideró estudiantes de secundaria entre las edades de 15 y 17 años, que desde una perspectiva evolutiva, comparten características musculoesqueléticas y de desarrollo comunes. Esto permite que la comparación entre los resultados obtenidos sea más factible ya que los los adolescentes pueden ser muy sensibles a las fuerzas de tracción ejercidas sobre ellos (Alexander, 1976; Arkin y Katz, 1956; Sward, 1992). 
Pocos estudios registran las tasas de incidencia de lesiones en función de las horas de exposición en EF o en otras actividades físicas en edad escolar. El presente estudio encontró que la tasa de incidencia era igual a 1,90 por cada 1.000 horas de exposición en las clases de educación física; 2,09 por cada 1.000 horas de actividad física organizada y 2,13 por cada 1.000 horas de actividad física no organizada. Estas cifras son más altas que las obtenidas por Verhanen y cols. $(2009)(0,50)$ en las clases de Educación Física, aunque la muestra para ese estudio tenía entre 10 y 12 años de edad. Al mismo tiempo, la incidencia encontrada en De Loes y cols. (1990) fue mayor $(3,20)$ para las clases de Educación Física en un grupo de edad similar (14-19 años) aunque estos autores solo consideraron el tiempo en las clases de EF. Spinks y cols. (2006) informaron una incidencia entre 1,19 y 2,18 por cada 1.000 horas, según el deporte.

Las lesiones más frecuentes que se producen durante la EF son lesiones musculares, seguidas por esguinces (específicamente en el tobillo) y contusiones, con porcentajes similares para cada tipo $(28,35 \%, 25,37 \%$ y $25,37 \%$, respectivamente). No hay unanimidad en los datos obtenidos en diferentes estudios. Algunos autores sugieren que los esguinces son las lesiones más comunes, como Nelson y cols. (2009), que registraron una incidencia del $40 \%$ frente al $19 \%$ para las lesiones musculares y por fractura. Carmeli y cols. (2003) registraron un porcentaje del $61 \%$ de lesiones debidas a esguinces y un $23 \%$ debido a fracturas, mientras que Sundblad y cols. (2005) registraron una incidencia del $48 \%$ de esguinces y lesiones musculares seguida por $14 \%$ de contusiones. Aun así, otros estudios han encontrado porcentajes más altos en lesiones de tipo muscular y contusiones (Backx et al., 1991; Grimaud et al., 2007).

Las lesiones más frecuentes originadas en las clases de Educación Física son las lesiones del miembro inferior $(73,13 \%)$ frente a las lesiones del miembro superior $(20,90 \%)$ y de cabeza y tronco $(5,97 \%)$. La mayoría de los resultados obtenidos por otros autores son consistentes con estos datos, aunque algunos han presentado porcentajes menores, que varían desde 39\% hasta $68 \%$ para lesiones de miembros inferiores (Backx et al., 1991; Carmeli et al., 2003; Grimaud et al. ., 2007; Nelson et al., 2009; Sundblad et al., 2005; Verhagen et al., 2009). En cuanto al mecanismo que originó las lesiones, se encontró que el traumatismo indirecto fue la causa más frecuente $(47,76 \%)$, seguido de traumatismo directo $(28,36 \%)$ y sobrecarga $(23,88 \%)$.

No se encontraron estudios que asociaran tipo de lesión, región anatómica y mecanismo de producción con falta de participación en las clases de E.F. El presente estudio mostró que los esguinces fueron la principal razón para no participar en las clases de educación física (35,65\%), seguidos de fracturas y tendinopatías, que reflejan la gravedad de estas lesiones en comparación con otras causas. Las personas con lesiones de los miembros inferiores acumulan una frecuencia de ausencias $(72,17 \%)$ muy superior a la de las personas con lesiones en los miembros superiores (22,61\%) y cabeza y tronco $(5,22 \%)$, pero simplemente porque ese porcentaje se corresponde con una mayor incidencia, no mayor gravedad. 
El porcentaje de lesiones que ocurren durante la Educación Física y no reciben tratamiento médico $(59,70 \%)$ es mayor que el porcentaje de lesiones que sí reciben tratamiento médico (40,30\%). Se hace evidente que estas últimas son más graves y se corresponden con mayor cantidad de ausencias durante las sesiones de clase. Estos resultados concuerdan con aquellos en la literatura científica que indican que del 40 al $50 \%$ de las lesiones en E.F. requieren atención médica y representan valores más bajos que las lesiones que ocurren fuera de las clases de E.F. (Backx et al., 1991; Grimaud et al., 2007).

\section{Limitaciones del estudio}

Como limitaciones de este estudio, se puede mencionar que aunque se aplicó un muestreo aleatorio, la población aún pertenece a una sola Comunidad (Madrid) y se recomienda extender el estudio a poblaciones más amplias. No ha habido oportunidad de encontrar un grupo de control apropiado ya que en España las clases de E.F. son obligatorias para este grupo de edad.

\section{CONCLUSIONES}

La ausencia en las clases de Educación Física debida a lesiones originadas en clase es muy baja $(0,3 \%)$ en comparación con la participación esperada de los estudiantes, por lo que no puede establecerse como una fuente significativa de no participación de los estudiantes en las sesiones de clase. La tasa de incidencia de lesiones en las clases de E.F. es 1,90 por cada 1.000 horas, que es menor que la tasa para la actividad física organizada y no organizada (2,09 y 2,13 , respectivamente).

La falta de participación en la clase de Educación Física se produce con mayor frecuencia por motivos ajenos a la salud, y entre aquellos que sí están relacionados con la salud, las faltas por enfermedad son más frecuentes que por lesión. Además, las lesiones fuera de las clases de Educación Física son más frecuentes que las que ocurren durante las clases. El tipo más frecuente de lesiones son las lesiones musculares, los esguinces y las contusiones, que suelen ser de menor gravedad que las tendinopatías y las fracturas, que requieren períodos más largos de inactividad como parte de su recuperación.

Las lesiones originadas en las clases de Educación Física se localizan con mayor frecuencia en los miembros inferiores y los traumatismos indirectos representan un porcentaje más elevado que las lesiones por traumatismo directo y sobrecargas. Sin embargo, con respecto a la acumulación de ausencias en la clase, no hay una diferencia significativa entre los distintos mecanismos de producción y regiones anatómicas. Las lesiones que no requieren atención médica son más frecuentes en la clase de Educación Física, sin diferencias significativas con respecto a las que sí lo requieren, mientras que son precisamente las lesiones que reciben tratamiento las que generan una proporción significativamente mayor de faltas de participación. 


\section{REFERENCIAS BIBLIOGRÁFICAS}

Abernethy, L., \& MacAuley, D. (2003). Impact of school sports injury. Br J Sports Med, 37(4), 354-355. https://doi.org/10.1136/bjsm.37.4.354 PMid:12893724 PMCid:PMC1724674

Alexander, C. J. (1976). Effect of growth rate on the strength of the growth plateshaft junction. Skeletal Radiology, 1, 67-76. https://doi.org/10.1007/BF00347411

Arkin, A. M., \& Katz, J. F. (1956). The effects of pressure on epiphyseal growth; the mechanism of plasticity of growing bone. J Bone Joint Surg Am, 38A(5), 1056-1076. https://doi.org/10.2106/00004623-195638050-00009

Backx, F. J., Beijer, H. J., Bol, E., \& Erich, W. B. (1991). Injuries in high-risk persons and high-risk sports. A longitudinal study of 1818 school children. Am J Sports Med, 19(2), 124-130. doi:10.1177/036354659101900206 https://doi.org/10.1177/036354659101900206

Belechri, M., Petridou, E., Kedikoglou, S., Trichopoulos, D., \& Sports Injuries European Union, G. (2001). Sports injuries among children in six European union countries. Eur J Epidemiol, 17(11), 1005-1012. https://doi.org/10.1023/A:1020078522493 PMid:12380712

Carmeli, E., Azencot, S., Wertheim, m., \& coleman, r. (2003). Sports injuries in students aged 12-18 during physical education classes in Israel. Biol Sport, 20, 271-280.

Carter, E. A., Westerman, B. J., \& Hunting, K. L. (2011). Risk of injury in basketball, football, and soccer players, ages 15 years and older, 20032007. J Athl Train, 46(5), 484-488. https://doi.org/10.4085/1062-605046.5.484 PMid:22488135 PMCid:PMC3418954

Collard, D. C., Chinapaw, M. J., van Mechelen, W., \& Verhagen, E. A. (2009). Design of the iPlay study: systematic development of a physical activity injury prevention programme for primary school children. Sports Med, 39(11), 889-901. doi:10.2165/11317880-000000000-00000 https://doi.org/10.2165/11317880-000000000-00000

Collard, D. C., Verhagen, E. A., van Mechelen, W., Heymans, M. W., \& Chinapaw, M. J. (2011). Economic burden of physical activity-related injuries in Dutch children aged 10-12. Br J Sports Med, 45(13), 1058-1063. doi:10.1136/bjsm.2010.082545 https://doi.org/10.1136/bjsm.2010.082545

de Loes, M., Jacobsson, B., \& Goldie, I. (1990). Risk exposure and incidence of injuries in school physical education at different activity levels. Can J Sport Sci, 15(2), 131-136. PMid:2383817

Emery, C. A. (2010). Injury prevention in paediatric sport-related injuries: a scientific approach. $\mathrm{Br} J$ Sports Med, 44(1), 64-69. doi:10.1136/bjsm.2009.068353 https://doi.org/10.1136/bjsm.2009.068353

Fuller, C. W., Ekstrand, J., Junge, A., Andersen, T. E., Bahr, R., Dvorak, J., . . Meeuwisse, W. H. (2006). Consensus statement on injury definitions and data collection procedures in studies of football (soccer) injuries. $\mathrm{Br} \mathrm{J}$ Sports Med, 40(3), 193-201. doi:10.1136/bjsm.2005.025270 https://doi.org/10.1136/bjsm.2005.025270

Grimaud, O., Piette, C., Clappier, P., Deguen, S., \& Pommier, J. (2007). [School sport injuries in the state schools of Ille-et-Vilaine (France)]. Arch Pediatr, 
14(4), 392-393. doi:10.1016/j.arcped.2007.01.011 https://doi.org/10.1016/j.arcped.2007.01.011

Gutiérrez-Castañón, E. (2014). Epidemiología de las lesiones deportivas en clase de Educación Física (Tesis doctoral). Madrid: Universidad Autónoma de Madrid.

Gutiérrez-Castañón, E. (2008). Lesiones y falta de actividad en Educación Física. Madrid: CV Ciencias del Deporte.

Gutiérrez-Castañón, E., Valbuena-Láiz, C., Álvarez-Barrio, M. J., Cid-Yagüe, L., Martínez-de-Haro, V., \& Muñoa-Blas, J. (2007). Causas de absentismo en Educación Física en ESO. Selección, 16(2), 84-90.

Habelt, S., Hasler, C. C., Steinbruck, K., \& Majewski, M. (2011). Sport injuries in adolescents. Orthop Rev (Pavia), 3(2), e18. doi:10.4081/or.2011.e18 https://doi.org/10.4081/or.2011.e18

Hagglund, M., Walden, M., Bahr, R., \& Ekstrand, J. (2005). Methods for epidemiological study of injuries to professional football players: developing the UEFA model. $\mathrm{Br} J$ Sports Med, 39(6), 340-346. doi:10.1136/bjsm.2005.018267 https://doi.org/10.1136/bjsm.2005.018267

Josse, J. M., MacKay, M., Osmond, M. H., \& MacPherson, A. K. (2009). School injury among Ottawa-area children: a population-based study. J Sch Health, 79(2), 45-50. doi:10.1111/j.1746-1561.2008.00375.x https://doi.org/10.1111/j.1746-1561.2008.00375.x

Lenaway, D. D., Ambler, A. G., \& Beaudoin, D. E. (1992). The epidemiology of school-related injuries: new perspectives. Am J Prev Med, 8(3), 193-198. https://doi.org/10.1016/S0749-3797(18)30831-6

Loder, R. T., \& Abrams, S. (2011). Temporal variation in childhood injury from common recreational activities. Injury, 42(9), 945-957. https://doi.org/10.1016/j.injury.2010.02.009 PMid:22081824

McKenzie, K., Fingerhut, L., Walker, S., Harrison, A., \& Harrison, J. E. (2012). Classifying external causes of injury: history, current approaches, and future directions. Epidemiol Rev, 34, 4-16. doi:10.1093/epirev/mxr014 https://doi.org/10.1093/epirev/mxr014

Nelson, N. G., Alhajj, M., Yard, E., Comstock, D., \& McKenzie, L. B. (2009). Physical education class injuries treated in emergency departments in the US in 1997-2007. Pediatrics, 124(3), 918-925. doi:10.1542/peds.20083843 https://doi.org/10.1542/peds.2008-3843

NOMESCO, N. M.-S. C. (2007). Classification of External Causes of Injuries. Fourth revised edition. Retrieved from https://norden.divaportal.org/smash/get/diva2:1201255/FULLTEXT01.pdf

Pakzad-Vaezi, K., \& Singhal, A. (2011). Trends in paediatric sport- and recreation-related injuries: An injury surveillance study at the British Columbia Children's Hospital (Vancouver, British Columbia) from 1992 to 2005. Paediatr Child Health, 16(4), 217-221. https://doi.org/10.1093/pch/16.4.217 PMid:22468125 PMCid:PMC3076173

Roach, R., \& Maffulli, N. (2003). Childhood injuries in sport. Physical Ther Sport, 4(2), 58-66. https://doi.org/10.1016/S1466-853X(02)00114-1

Sosnowska, S., \& Kostka, T. (2003). Epidemiology of school accidents during a six school-year period in one region in Poland. Eur J Epidemiol, 18(10), 977-982. https://doi.org/10.1023/A:1025802203726 PMid:14598928 
Spinks, A. B., Macpherson, A. K., Bain, C., \& McClure, R. J. (2006). Injury risk from popular childhood physical activities: results from an Australian primary school cohort. Inj Prev, 12(6), 390-394. doi:10.1136/ip.2006.011502 https://doi.org/10.1136/ip.2006.011502

Sundblad, G., Saartok, T., Engstrom, L. M., \& Renstrom, P. (2005). Injuries during physical activity in school children. Scand J Med Sci Sports, 15(5), 313323. doi:10.1111/j.1600-0838.2004.00419.x https://doi.org/10.1111/j.1600-0838.2004.00419.x

Sward, L. (1992). The thoracolumbar spine in young elite athletes. Current concepts on the effects of physical training. Sports Med, 13(5), 357-364. https://doi.org/10.2165/00007256-199213050-00005

Taylor, B. L., \& Attia, M. W. (2000). Sports-related injuries in children. Acad Emerg Med, $7(12)$ 1376-1382. https://doi.org/10.1111/j.1553-2712.2000.tb00495.x PMid:11099428

Verhagen, E., Collard, D., Paw, M. C., \& van Mechelen, W. (2009). A prospective cohort study on physical activity and sports-related injuries in 10-12-yearold children. $\mathrm{Br} J$ Sports Med, 43(13), 1031-1035. doi:10.1136/bjsm.2008.055483 https://doi.org/10.1136/bjsm.2008.055483

Videmsek, M., Karpljuk, D., Mlinar, S., Mesko, M., \& Stihec, J. (2010). Injuries to primary school pupils and secondary school students during physical education classes and in their leisure time. Coll Antropol, 34(3), 973-980. PMid:20977091

Número de citas totales / Total references: 30 (100\%)

Número de citas propias de la revista / Journal's own references: $0(0 \%)$

Rev.int.med.cienc.act.fís.deporte - vol. 18 - número 72 - ISSN: 1577-0354 